\title{
4-State Rotary Joint Control: Results With a Novel Programmable Brake
}

\author{
Yaroslav Tenzer, IEEE Student Member, Brian L Davies, Ferdinando Rodriguez y Baena, IEEE, Member \\ Department of Mechanical Engineering, Imperial College London, London SW7 2AZ, UK
}

\begin{abstract}
This paper outlines the development of a new programmable rotary brake which can restrict motion in one direction while allowing free rotation in the opposite direction. The design, implementation and performance of a fully-functional prototype are described, followed by the development of control schemes which allow different operational modes. A number of unique performance parameters for the brake are identified and analytical methods for their evaluation are proposed. The prototype, which was developed using commercially-available miniature brakes, has a cylindrical shape and is characterized by a compact, modular and cost-effective design which can be implemented into man-machine interfaces to restrict undesired user motion without the need for an explicit force sensor. The programmable brake concept proposed here is advantageous in these applications, where close cooperation with humans poses significant safety risks, due to the inherent safety of a mechanism which is not able to move autonomously in the event of a malfunction.
\end{abstract}

Index Terms-rotary joint, programmable brake, passive haptics, robotics, constraints generation.

\section{INTRODUCTION}

A design philosophy based on passive components such as brakes and clutches is relevant to the development of many man-machine interfaces, in particular those in which the robot works in close proximity or hand-in-hand with the human operator. Such applications range from surgery [6], [21], rehabilitation [18], haptics for training and diagnostics [8] to complex mechanical assembly [2], and many other applications for which human safety is critical. These assisting robots are also called "collaboration robots" or cobots [5]. They are designed to provide assistance to the human operator while being intrinsically passive. Robotic passivity can be rigorously defined by the direction of the power flow between the mechanism and the environment with which it interacts [14]. Intuitively, passivity can be seen as a robot being programmed to react rather than to act. Alternatively, the robot may be passive by design thus preventing rather than initiating motion. Robots which belong to the former category use active components such as motors and as a result can pose a safety risk during a malfunction. By contrast, robots belonging to the latter category use passive components, such as brakes, to dissipate or redirect energy, which results in an inherently safer system.

Different types of brakes and clutches have been used in human-machine applications, for instance: electromagnetic brakes [7], magnetic particle brakes [20], piezoelectric actuated brakes [11], wafer disk brakes [9], shoe-type brakes [24], eddy-current based brakes [12] and magneto-rheological brakes [17], [4]. These brakes are differentiated in terms of performance characteristics such as reaction torque, activation time and power consumption. However, when activated, such brakes limit or even prevent motion in all directions, for example clockwise and anticlockwise directions in rotary configurations. The unlocking algorithm therefore requires an additional sensor to determine when user-applied forces are in a safe or desired- direction. For example, in some devices featuring brakes, it is common to have a force sensor integrated into the mechanism ([7],[25],[16]) in addition to joint encoders, which are used to monitor joint positions. The force sensor allows the direction and magnitude of the user's applied force to be sensed and the brakes to be engaged or disengaged depending on the configuration of the required restrictions. An advantage of this system design is that, as well as providing programmable constraints, it is able to produce the high reaction forces required in some applications. The integration of a locking mechanism, however, comes at a price: a force sensor must be used and this adds to the size and complexity of the device. The resulting system therefore not only has a complicated architecture but may also suffer from undesired effects. For example, in force-feedback devices, the transient reaction forces which occur as a result of mechanical latency (i.e. deactivation time) are felt by users as "sticking". Although these forces last just a few milliseconds they can be perceived by a human operator, since the nervous system is capable of detecting force transients with frequencies of up to $1 \mathrm{kHz}$ [3].

An alternative to the "brake-force sensor concept" is offered by "part-locking" mechanisms, where the movement of the end-effector is restricted physically in one direction while allowing movement in other directions. One solution would be to introduce some play between the link shaft and brake housing, which could be detected by a suitably mounted position sensor [19]. In the event of the brake being fully engaged, detection of a finite motion in either direction could serve as the signal to release the brake. . This approach is suitable for some applications, such as a car-user interface [13], but it is unsuitable for applications where a full locking of one of the joints may be required, which a- mechanism with a "play" is unable to provide.

Alternatively, part-locking can be achieved by mounting a spring between the brake and the shaft [10]. Therefore, when an external torque is applied to the shaft and the brake is activated, the torque would work against the spring and the reaction torque would be dependent on its stiffness. This feature and the ability of preloaded springs to keep the 
reaction forces in a static configuration allows simulation of elastic deformations, which is impossible with a brake alone. An external torque applied on the shaft would result in its rotation, therefore shaft rotation monitoring using an encoder is possible; as in the previous example this eliminates the need for a force sensor, but means that full shaft locking becomes impossible.

A third approach to mechanical part-locking uses physical stoppers, and is presented in [15]. In this embodiment, shaft rotation is limited within boundaries created by physical stoppers. The stoppers are solid parts mounted on the axis of the shaft and their angular orientation is controlled using dedicated rotary actuators. By adjusting the angular orientation of the stoppers, the rotation boundaries can be adjusted. The advantage of this concept is in its ability to create high reaction forces, while rotation in the opposite direction, away from the stopper, is still allowed. The mechanism, however, is complex and costly due to the need for two additional motors to orient the stoppers at every joint.

Finally, mechanical part-locking can be achieved by customdeveloped mechanical joints based on the "freewheel" mechanism. In this case the locking mechanism includes special disks with guiding slots filled with metal balls. Shaft rotation is prevented in one direction because the balls are wedged between tapered walls, while rotation in the other direction is not restricted because the balls fit into dedicated gaps. By using two locking parts which mirror each other, and controlling the rotation speed of the outer wall, it is possible to configure joint restrictions into 4 independent states: free motion in both directions, constrained motion in one direction (clockwise or counterclockwise) or no motion at all. A truly passive robot built using this technology was the PADyC (Passive Arm with Dynamic Constraints), which allows constrained motion of a tool with respect to a pre-planned task in the context of robot assisted surgery [21]. A clear advantage of the PADyC system is that it can limit the rotating speed of the joints and therefore guide the end effector in a particular trajectory, while being incapable of any autonomous motion. However, the implementation of this technology is highly complex due to the use of "freewheels" and two electrical motors per every joint.

This paper discusses the novel concept of a programmable brake implementing stoppers, but without the shortcomings of the design presented in [15]. The proposed programmable brake falls within the part-locking mechanism family and was first presented in [23], in which results on a 2 DOF system incorporating prototypes of these brakes successfully demonstrated the viability of the concept. This paper describes the work which was carried out to model the characteristics of the brake and further develop the concept into a fully functional modular prototype suitable for integration into any number of mechanical systems. A novel design is proposed which is more compact, operates faster, locks more firmly and uses less energy than the prototype presented in [23]. In addition, different control schemes are presented here and the limitations and shortcomings of the programmable brake mechanism are analyzed. The paper is organized as follows: the mechanism concept is briefly introduced in Section II-A,
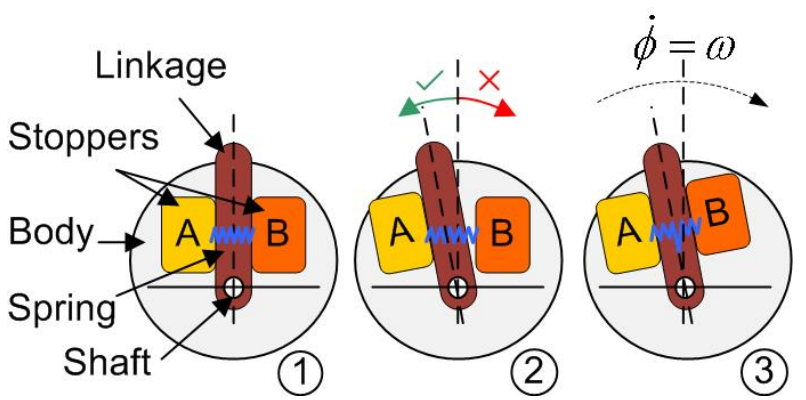

Figure 1: Programmable brake concept. (1) Static configuration. (2) Shaft turning away from the restriction. (3) Stopper $\mathrm{B}$ is deactivated and pulled by the spring

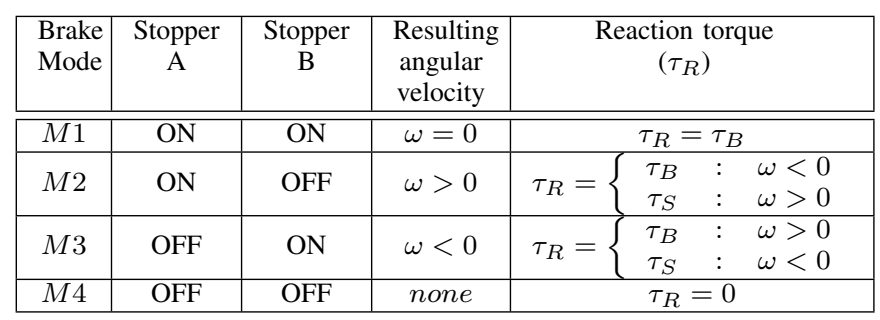

Table I: Possible locking modes for the programmable brake. $\tau_{R}$ - the resulting reaction torque, $\tau_{B^{-}}$the maximum brake friction torque, $\tau_{S}$ - the reaction torque produced by the preloaded spring.

followed by a description of the novel design in Section II-B and the definition of viable control schemes in Section II-C. The performance characterization of the prototype (Section III) is carried out by analyzing the performance of the individual components, estimating mechanical limitations and developing numerical models for validation. Summary and conclusions are included in Section IV.

\section{The Programmable BRAKe Unit}

\section{A. Brake Concept}

The novel concept of a part-locking brake mechanism was first presented in [23] and is illustrated in Fig. 1, in a rotary configuration. The linkage is connected to the shaft such that they rotate in tandem. The angular position of the shaft is accurately measured by means of a suitable position sensor (e.g. a potentiometer or optical encoder). Two stoppers (A and $\mathrm{B}$ ) are positioned one on either side of the linkage and pulled flat to the linkage by a preloaded elastic spring. The stoppers can be activated separately using a clutch or a brake device, resulting in a mechanical engagement, such as frictional engagement, with the body. The activated stopper then restricts the rotation of the linkage in the direction of the stopper. The deactivation of the stopper is performed according to a pre-programmed logic. For example, if a restriction in the clockwise direction is required (Fig. 1.2), then stopper B is activated and this prevents the rotation of the shaft in the direction of the stopper, but the shaft is still free to rotate in the opposite direction. Rotation of the shaft in the opposite direction is recognized using the attached position sensor and as soon as rotation is detected, stopper B is deactivated by a 
pre-programmed algorithm. This releases stopper B from the body of the brake, which then causes the preloaded spring to pull the stopper back flush to the link, ready for the next constraint command. By activating the stoppers in different sequences, a number of configuration modes are possible, as summarized in Table I, where $M 1-M 4$ represent the four configuration modes, $\omega$ is the resulting angular velocity restriction and $\tau_{R}$ is the resulting reaction torque, which is either the the maximum friction torque produced by the brake $\tau_{B}$ or the reaction torque produced by the preloaded spring $\tau_{S}$.

\section{B. Brake Design}

A prototype of the brake was manufactured-according to the exploded design illustrated in Fig. 2. The programmable brake unit comprises a shaft (1) and a linkage (2), which are joined with a screw and are free to rotate as one unit relative to the body (3). The linkage is held between two stoppers (4), and positioned contiguously to them. Each stopper unit consists of the stopper part and a miniature commercial electromagnetic brake (Part number: 14.110.03.103, Magneta[1]). Electromagnetic brakes were chosen for this prototype because they provide high reaction torque per unit mass, are simple to operate and are cheap when compared to other brake types (Sec. I). The miniature brake consists of an armature plate (5) and a stator (6). The plate is free to rotate relative to the shaft while the stator is fixed to the body. The armature plates are connected to the stoppers through leaf springs (not shown), thus the rotational force is fully transmitted while a small axial motion is permitted. Some axial motion is required to allow movement ( $1 \mathrm{~mm}$ gap [1]) of the armature plate toward the stator during activation, due to the magnetic field resulting from electric current running inside the brake coil. This movement causes frictional engagement between the armature plate and the stator, preventing further rotation of the plate. The armature plate will be pulled back by the leaf springs when the brake is switched off. The cover plates (7) are designed to hold the parts together, house low-friction ball bearings to support the shaft and allow mounting for the encoder (8) which senses the rotation of the shaft. A spring, not shown in Fig. 2 but visible in Fig.3, is fixed to the two stoppers and passes through the linkage freely. The spring is preloaded, which puts initial force on the stoppers and keeps them in a "standby position", flush on either side of the linkage itself.

Finally, a safety mechanism to prevent spring overstretching is implemented as part of the stoppers assembly. A "play mechanism" keeps the motion of the linkage within hard limits: in the event of one stopper being activated, movement away from the stopper is not restricted till the safety gap is reached, after which point the linkage collides with the fixed stopper, preventing further motion, as illustrated in Fig. 4.

\section{Brake Control}

As described in II-A, the novel programmable brake unit has a number of possible configuration modes and a control algorithm is required to operate the brake and switch between

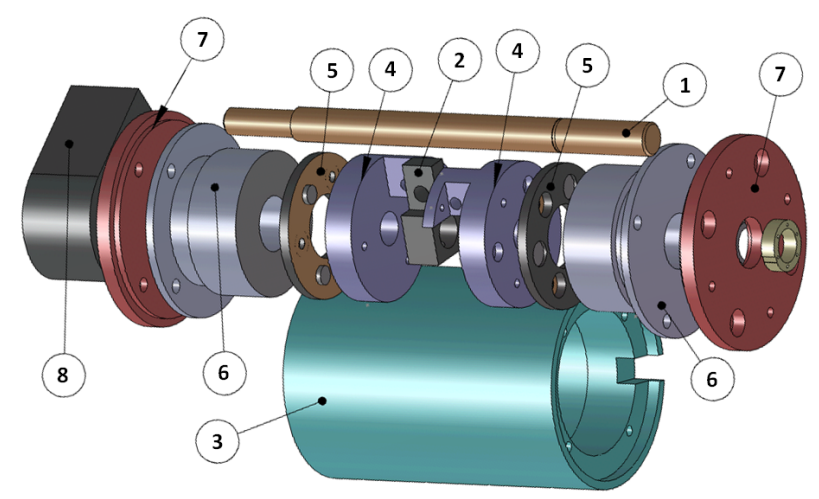

(a)

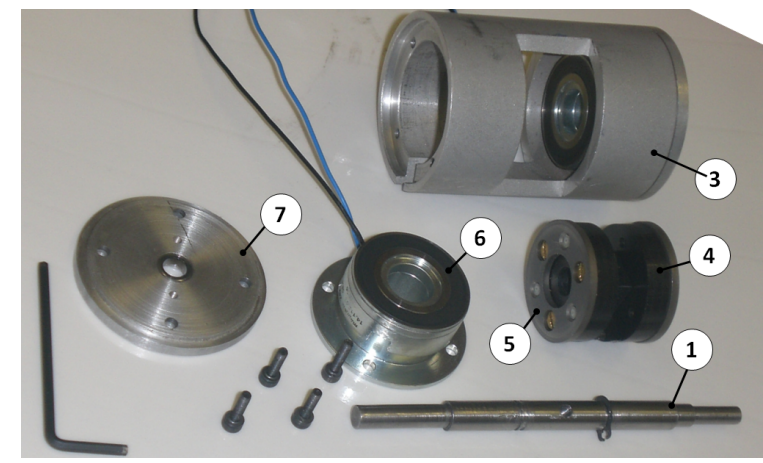

(b)

Figure 2: Programmable brake of cylindrical type in exploded view.

(1) shaft, (2) linkage, (3) body, (4) stopper A and B, (5) armature friction plate, (6) commercial magnetic brakes, (7) cover plate, (8) encoder
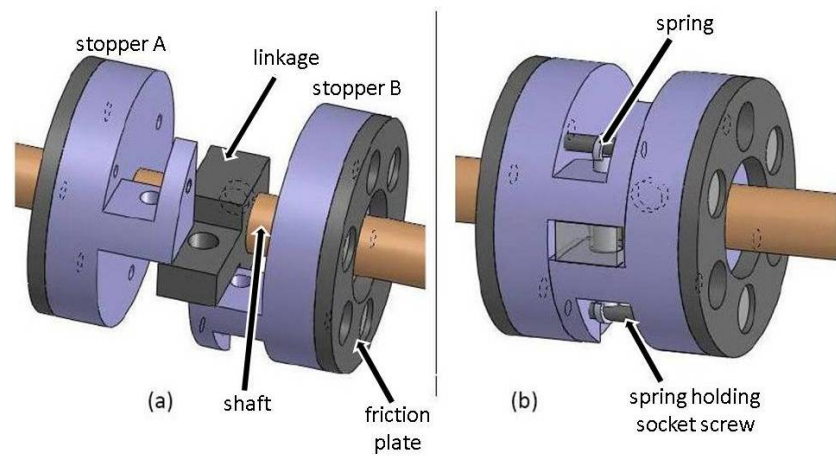

Figure 3: The stoppers and the spring arrangement inside the programmable brake. (a) Exploded view. (b) Fully assembled mechanism. 

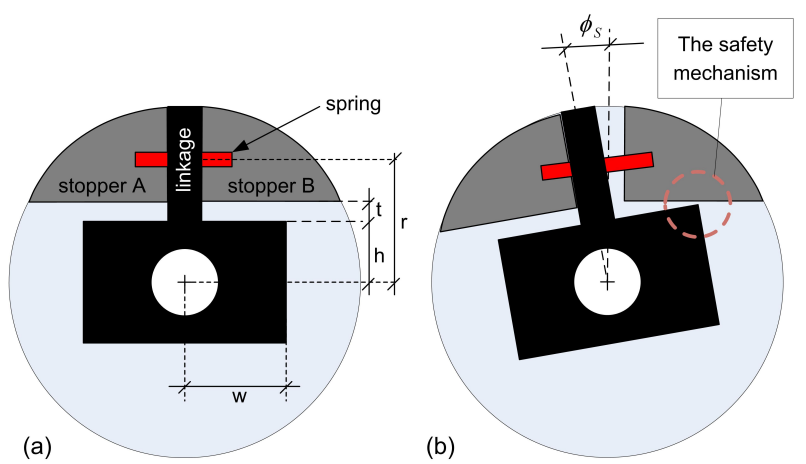

Figure 4: Safety gap explained. (a) Static configuration where stopper B is activated. (b) Shaft rotation till safety gap $t$ is closed.

them. The brake shaft can be switched into one of the following configuration modes: no restriction (M4), fully-locked (M1) or part-locked (M2/M3), see Table I. No restriction is achieved by switching stoppers A and B OFF and fully-locked is achieved by switching both stoppers ON (as in Fig. 1). The part-locked uni-directional configuration can be achieved by switching stopper A ON and B OFF (or vice versa). Once in the part-locked situation, correct brake operation requires reconfiguration of the stoppers to maintain the restriction while enabling motion in the unconstrained direction, which can be achieved by applying one of the following control schemes:

Velocity scheme: It restricts the rotation of the shaft in one direction but allows motion in the opposite direction, similar to a "ratchet" mechanism. In this mode, the control algorithm monitors the velocity of the shaft and activates the stoppers when the shaft velocity is zero to restrict rotation in the undesired direction, then switches them off when rotation in the allowed direction is detected. The operational mode of the brake is a function of the required angular velocity restriction $\omega_{B}$ and the monitored shaft angular velocity $\omega$ (clockwise rotation is positive), and can be summarized as follows:

$$
M=\left\{\begin{array}{c}
M 2: \quad \omega \leq 0, \omega_{B}>0 \\
M 4: \quad \omega>0, \omega_{B}>0 \\
\hline M 3: \quad \omega \geq 0, \omega_{B}<0 \\
M 4: \quad \omega<0, \omega_{B}<0
\end{array}\right.
$$

The advantage of this control scheme is that rotation of the shaft in the undesired direction is restricted while the shaft is free to turn in the opposite direction. The disadvantage is that reactivation of the brake may occur during the stopping transient, when the shaft acceleration is above the critical value (as discussed in Section III-B), at which point the linkage would be stopped by the safety mechanism, as explained in Section II-B. This can be resolved by applying a time delay before reactivation.

Position scheme: It restricts the rotation of the shaft when the shaft angle becomes larger or smaller than a predefined value. In this mode, the control algorithm monitors the shaft angle $\phi$ and activates the relevant stopper once it crosses the restricted angle $\phi_{R}$. In mathematical form:

$$
M=\left\{\begin{array}{ccc}
M 4 & : & \phi<\phi_{R} \\
M 3 & : & \phi \geq \phi_{R} \\
\hline M 4 & : & \phi_{R}<\phi \\
M 2 & : & \phi_{R} \geq \phi
\end{array}\right.
$$

The advantage of this scheme is that it restricts the rotation of the shaft within an area by only monitoring the rotation angle of the shaft. However, was shaft rotation to be fast enough to cause a penetration error (i.e. stopping to occur slightly within the restricted area due to e.g. finite sampling time), a small movement away from the activated stopper may not be sufficient to trigger a release of the brake. To overcome this limitation, both the position and velocity of the shaft should be monitored, as described in the following section.

Advanced position scheme: It restricts the rotation of the shaft when the shaft angle becomes larger or smaller than a predefined value and uses the velocity of the shaft for deactivation purposes. This logic can be summarized using the following equations:

$$
M=\left\{\begin{array}{ccc}
M 4 & : & \omega<0 \bigvee \phi<\phi_{R} \\
M 3 & : & \phi \geq \phi_{R} \\
\hline M 4 & : & \omega>0 \bigvee \phi_{R}<\phi \\
M 2 & : & \phi_{R} \geq \phi
\end{array}\right.
$$

In this scheme, by monitoring the shaft velocity, the controller would disengage the stoppers when the shaft turns in the direction of the unrestricted zone, irrespective of whether a penetration error has occurred.

\section{Prototype Characterization}

A prototype of the programmable rotary brake described in Section II was developed and tested to assess its performance, then a simulation model based on its performance characteristics was developed to validate critical performance parameters. Details of this work are presented in the following sections.

\section{A. Activation and Deactivation}

The programmable brake mechanism uses miniature commercial electromagnetic brakes (Section II-B) to activate the stoppers. The specifications of the miniature brakes are described in the manufacturer datasheet, but performance may vary when the brake is integrated into a custom mechanism with bespoke control electronics. A number of experiments were thus performed to estimate the maximum braking torque and the activation and the deactivation time. These experiments focused on the performance of a single component, but were performed on a fully assembled mechanism.

The measurement of the maximum braking torque was performed in the following manner. A linkage was attached to the shaft and its orientation was horizontally fixed by activating the bottom stopper. A varying mass was applied at the endpoint of the horizontally oriented linkage till a slippage of the shaft occurred. A constant current source with a magnitude of $0.21 A$, provided by a standard commercial power supply (PS5005, Vellman Instruments), was used to keep the magnetic forces constant. The maximum torque which could be applied 
to the joint before any slippage occurred was found to be $0.6 \mathrm{Nm}$.

The response time of the brake was also measured to evaluate its activation and deactivation time in response to a control signal. When the brake is actuated, the armature plate is attracted to the stator and the air-gap between them is closed, which creates a small transient vibration that can be detected. The activation time was thus measured using the following procedure; the brake unit was resting, without a load applied to the shaft, with an accelerometer (353B03 PCB Piezotronics Inc.) magnetically attached to the body of the brake. A custom program was written in LabView (The National Instruments Corporation) to record the control signal and the vibration signal at $4 k H z$ via a commercial USB data acquisition card (NI USB 6009). The samples were analyzed using Matlab (The MathWorks Inc.) and the "activation time" was extracted as the delay between the voltage raise on the coil and the maximum peak of the vibration signal, which occurs at the point of contact between the plate and the stator.- The average activation time of ten experiments, which was found to be normally distributed, was found to be $4.25 \mathrm{~ms}(226 \mathrm{~Hz})$, with a standard deviation of $0.54 \mathrm{~ms}$.

The brake's deactivation time was measured with an an external torque applied to the shaft and without an external load. The measurement without an external load corresponds to the maximum value for the deactivation time. It was measured similarly to the activation time experiment, the deactivation time was extracted as the delay between the voltage drop on the coil and the maximum peak of the vibration signal, which is created by the armature plate as it detaches from the stator and comes to its stand-by position on the rotor. The average deactivation time, from ten experiments, was found to be normally distributed, with a mean value of $36 \mathrm{~ms}(27.8 \mathrm{~Hz})$ and a standard deviation of $4.6 \mathrm{~ms}$. This result is higher than that reported in the brake datasheet [1], which is $4 \mathrm{~ms}$ for $90 \%$ torque reduction and not for full deactivation. The additional delay may be due to the increased inertia of the programmable brake setup, which may delay motion by a few milliseconds, and the lack of a current drain to actively de-energize the brake on switch off. A more appropriate current source will be investigated in the course of future work, as it does not impact upon the conclusions of this work.

Finally, the brake's deactivation time as a function of an externally applied torque was measured using the following procedure; the brake was activated and a variable weight was applied manually at the endpoint of a linkage connected to the shaft. A custom program was written in $\mathrm{C}++$ to record the current status of the brake and the shaft position, available through an encoder (HEDS-5500\#A06 by Hewlett Packard) mounted on the brake shaft. The program sampled the values at $1 \mathrm{kHz}$ via a commercial data acquisition card (NextMove2 by Baldor Inc), which were analyzed using Matlab. The deactivation time was extracted as the delay between the voltage drop on the coil and a measured change in shaft rotation. As expected, the resulting relation (represented as a solid line in Fig. 5) is approximately exponential in shape $(r=0.98)$ due to the fact that the current in the coil decays exponentially after the voltage is switched off. The expected

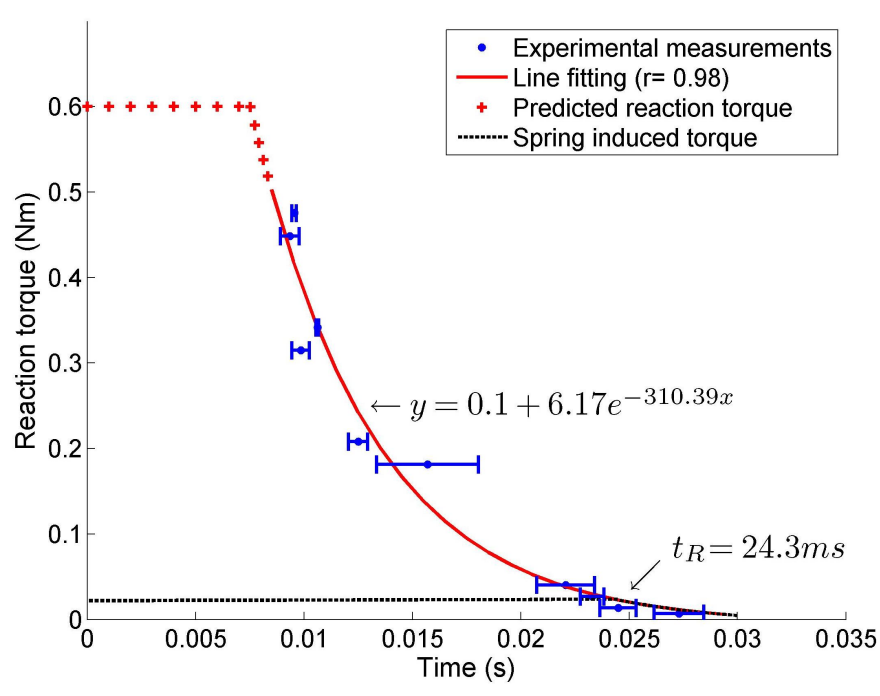

Figure 5: Brake deactivation time as a function of the applied torque

reaction torque between switch off and the start of the decay is represented by the dashed extention of the solid line and represents the short delay introduced by the inertia of the shaft-mounted linkage. As the external torque overcomes the friction torque, shaft rotation occurs and this is detected using the encoder. The influence of ambient temperature and relative humidity, which have been shown to affect brake performance [22], were not considered in this work.

\section{B. Mechanical Performance Estimation}

A number of performance characteristics were identified as important for the novel mechanism and are presented in the following section. The first is the reaction torque produced by the elastic spring (Fig. 3), which is designed to bring the stopper back into alignment with the linkage after deactivation. In the current prototype a linear spring was used and its spring constant was found experimentally to be $k=181.47 \mathrm{~N} / \mathrm{m}$. The reaction torque $\left(\tau_{S}\right)$ can be calculated from the kinematics of the prototype (Fig 4) using the following equation:

$$
\tau_{S}=k\left(\phi+\phi_{P}\right) r^{2} \quad: \quad 0 \leq \phi \leq \phi_{S}
$$

where $k$ is the spring constant, $r$ is the radius at which the spring is mounted, $\phi$ is the angle between the linkage and the stopper, $\phi_{P}$ is due to the initial pre-loading of the spring and the $\phi_{S}$ is the maximum safety angle $\phi$ can turn when the stopper is activated. The safety angle is a function of the safety mechanism implemented to avoid over stretching of the spring (Section II-B), which is schematically illustrated in Fig. 4. The gap $t$ between the linkage and the stopper was adjusted by choosing appropriate values for linkage parameters $(\mathrm{h}, \mathrm{w})$, which result in a maximum angle $\phi_{S}$ before the linkage collides with the stationary stopper. The extension of the spring was calculated as the length of an ark as a function of the angle, though a linear spring is used in this embodiment. This approximation was applied because the angular displacement $\phi$ is restricted by the safety angle $\phi_{S}$, which is small for this 
prototype $\left(\phi_{S}=5.2^{\circ}\right)$. The spring pre-load angle $\phi_{P}$ was estimated from the spring's reaction force and found to be correspond to $70^{\circ}$ at $r=10 \mathrm{~mm}$. The reaction torque was found to be between $22.1 \mathrm{mNm}$ and $23.8 \mathrm{mNm}$ for $\phi=0^{\circ}$ and $\phi=5.2^{\circ}$ respectively.

The second performance characteristic is the "critical shaft acceleration" $\alpha_{\text {critical }}$ for which the shaft would reach the critical safety angle at the same time as full deactivation of the stopper. The safety angle, as described earlier, is designed to mechanically limit the rotation of the shaft and prevent any over-stretching of the spring. Therefore, if the shaft accelerates above $\alpha_{\text {critical }}$, it would reach the safety angle before the stopper deactivates and therefore the shaft would be stopped. The critical angular acceleration of the shaft can be calculated as follows:

$$
\alpha_{\text {critical }}=\frac{2 \phi_{S}}{\left(t_{B}+1 / f_{C}+t_{S}\right)^{2}}
$$

Where $t_{B}$ is the deactivation time of the stopper, $f_{C}$ is the frequency of the control loop, $t_{S}$ captures any latency during the aquisition and processing of position measurements, and $\phi_{S}$ is the safety angle. The period of the control algorithm $\left(1 / f_{C}\right)$ and the latency due to the sensors $\left(t_{S}\right)$ have been included to account for the reaction time of the system, which may vary from setup to setup. The shaft accelerations at the critical value and above are schematically represented in Fig. 6. The shaded rectangular region represents the critical area where the " $x$ " intercept coincides with the sum of the deactivation time of the magnetic brake and the latency of the system $\left(t_{B}+1 / f_{C}+t_{S}\right)$ and the "y" intercept coincides with the value of the mechanical safety gap $\left(\phi_{S}\right)$. The dashed line represents shaft rotation at the critical acceleration. In this case, the shaft turns against the spring (areas A, B and C) and is free to move as soon as the brake is deactivated (area D). The solid line represents shaft rotation at acceleration for accelerations above $\alpha_{\text {critical }}$. When the shaft accelerates above the critical value, it reaches the critical angle $\left(\phi=\phi_{S}\right)$ before the stopper deactivates and therefore the shaft is stopped (area B). The shaft will remain still until the external turning torque overcomes the decaying brake torque (area $\mathrm{C}$ ), before accelerating once again (area D).

The relationship between an externally applied torque and the programmable brake's reaction torque $\left(\tau_{S}\right)$ represents one of the unique features of this brake design, which virtually eliminates the sticking sensation experienced by users of alternative brake-based systems. Indeed, Fig. 5 displays the reaction torque due to the spring at a critical acceleration superimposed to the measured deactivation response of the electromagnetic brake used in these experiments. As can be seen, the reaction torque due to the spring is much lower than the friction induced torque of the brake until the two lines intersect at $24.3 \mathrm{~ms}$. This intersection, marked as $t_{R}$, coincides with the moment at which the decaying brake torque equals the reaction torque due to the stretching spring. From this point onwards the friction of the brake would be lower than the reaction due to the spring, causing the two responses to converge to the same trend thereafter. In Eq. 5, the deactivation of the brake $t_{B}$ can potentially be replaced by $t_{R}$, which

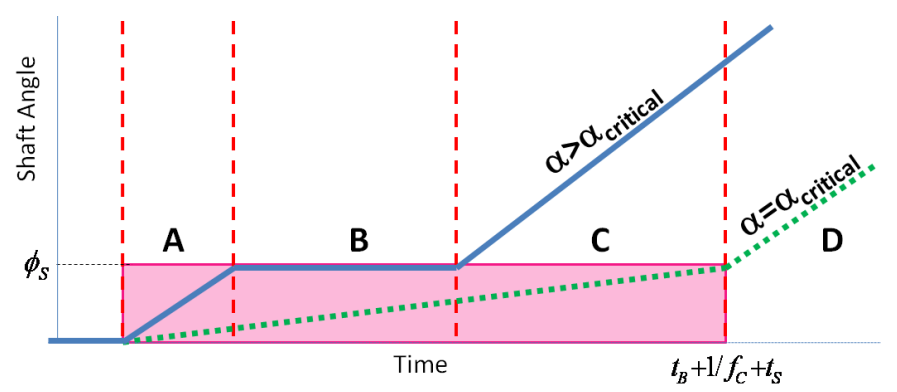

Figure 6: Unlocking area explained

\begin{tabular}{|c|c|}
\hline Calculated brake parameters & Value \\
\hline Brake inertia & $7.26 \mathrm{e}-7 \mathrm{~kg} \cdot \mathrm{m}^{2}$ \\
\hline Stopper inertia & $3.61 \mathrm{e}-7 \mathrm{~kg} \cdot \mathrm{m}^{2}$ \\
\hline Brake rotational spring k & $0.174 \mathrm{Nm} / \mathrm{rad}$ \\
\hline Rotational spring pre-load & $70 \mathrm{deg}$ \\
\hline Rotational friction (Coulomb) & $0.00049 \mathrm{Nm}$ \\
\hline Hard stop gap & $5.2 \mathrm{deg}$ \\
\hline $\begin{array}{c}\text { Motor parameters 3257024CR by Faulhaber } \\
\text { (from [1]) }\end{array}$ & Value \\
\hline Inertia & $41 \mathrm{e}-7 \mathrm{~kg} \cdot \mathrm{m}^{2}$ \\
\hline Torque constant & $0.0377 \mathrm{Nm} / \mathrm{A}$ \\
\hline
\end{tabular}

Table II: Simulation parameters

is the timing at which the reaction force due to the spring is equal to the reaction force due to friction. Nevertheless, practically finding $t_{R}$ would be difficult in other applications so $t_{B}$ was used here, an overestimation that may result in a higher maximum acceleration, which could be significant.

\section{Modelling}

To estimate the critical performance characteristics of the novel brake, a simulation model was developed. The simulation was prepared in Simulink vR2009b (Mathworks Inc.) using Simscape building blocks. The model-specific parameters used in the simulation are summarized in Table II. Some of these parameters were obtained through manufacturer datasheets, while others were estimated from simple experiments on the prototype. Based on the mechanical design of the brake, described in section II-B, the model blocks can be summarized as follows: the inertia of the linkage together with the inertia of the shaft are separated from the inertia of the magnetic stopper by a rotary spring, a friction block and a double sided hard stop. The hard stop limits the rotation of the linkage relative to the stopper within the safety gap. The brake is modelled against the industrial miniature electromagnetic brake used in the prototype (Sec.II-B) using a Karnopp stickslip model, similar to [22]. The friction of the shaft was not modelled in this simulation as the shaft is mounted on low-friction ball bearings of neglegible friction. It should be noted that the mechanism was simulated with only one stopper because the unlocking for the second stopper is identical. Also, the delay introduced by a discrete controller and the sensor was neglected because the deactivation time $\left(t_{B}\right)$ was assumed to be much higher then the control refresh period and sensor latency $\left(1 / f_{C}+t_{S}\right)$. 


\section{Model Validation}

To validate the simulation model, a test rig was built with the programmable brake direct driven by a DC motor (3257024CR by FAULHABER) with an attached encoder (HEDS-5500\#A06 by Hewlett Packard). The brake, motor and encoder were controlled with an embedded control board (Next Move PCI2 by Baldor Inc.). A custom-developed current driver was positioned between the control board and the brake, to amplify the control signal. Conversely, a commercial current driver (ADS_E 50/5 by Maxon Inc.) was connected between the control board and the motor. A control algorithm, to control activation and deactivation, was developed in embedded $\mathrm{C}$ and executed on the embedded card at a speed of $5 k \mathrm{~Hz}$. The algorithm followed the velocity scheme logic (Sec. II-C) and switched the stopper off as soon as rotation away from the stopper was detected. The current across the brake was adjusted manually to $0.21 A$ to maintain agreement with previous experiments.

A series of tests with different external torques, applied by the motor on the brake, were performed. At the start of each experiment no torque was applied, the shaft was at zero velocity and a stopper was activated. A step command was then sent to the motor, which turned the shaft in the opposite direction to the activated stopper, followed by a signal to deactivate the stoppers, which was triggered by shaft motion detected by the encoder (i.e. velocity control scheme). The position of the shaft and the status of the brake were sampled and recorded on a standard PC using customdeveloped software at a speed of $1 \mathrm{kHz}$.

For every experiment, the motor power was adjusted and the following ratios were used: $100 \%$ (or 50W), 60\%, 20\% and $10 \%$ (or $0.5 \mathrm{~W}$ ). Each experiment was repeated ten times for statistical reproducibility.

\section{E. Results and Discussion}

Experimental and simulated results for the acceleration of the shaft for different power ratios are summarized in Fig. 7. Given the reproducibility of measurements, the experimental results were expected to lie within the shaded dashed band shown, which corresponds to the angular shaft position at a given power level over ten repetitions. As can be observed, $100 \%$ and $60 \%$ motor power resulted in accelerations above $\alpha_{\text {critical }}$, resulting in a temporary stoppage of the shaft due to the mechanical safety mechanism. Also, at $100 \%$ power it can be seen that the transient stoppage occurs above the safety angle; this can be explained by the inability of the stopper to withstand the shaft impact, thus causing a finite slip between the stator and rotor friction plates of the brake. At $20 \%$ motor power, it can be seen that the transient stoppage occurred in the safety area, which indicates that the motor was not powerful enough to overcome the resistance induced by the stretching spring. Interestingly, the simulation at $20 \%$ power shows a similar acceleration trend, but without the transient stoppage. This can be explained by looking closely at the experimental setup; the motor is directly connected to the brake, which results in a highly sensitive system in which small variations in current amplifier, motor performance and friction have a profound impact. These variations influence the shaft acceleration, but cannot be simulated using simple tools. Finally, the experimental results at $10 \%$ of the motor power correlate well with those of the simulation. It is clear that the motor at this power ratio is not strong enough to overcome the resistance of the spring and friction, which explains why no shaft turning occurs until the brake is fully deactivated. Though Fig. 7 shows discrepancies within the safety zone between the experimental and the simulated results, these are relatively small and their impact in applications such as haptics is expected to be negligible. In addition, experimental results at $20 \%$ power do not correlate well with those produced in simulation, however they corroborate the trends derived in previous sections, which is of interest as a means of determining the boundaries of the safe area. The critical acceleration of the shaft was estimated as $148.2 \mathrm{rad} / \mathrm{s}^{2}$ using Eq. 5, and is also presented in Fig. 7. It is important to note that, though the critical acceleration of the shaft- is a performance parameter of the programmable brake, it was difficult to achieve constant acceleration during the experiments due to non-linearities in the motor performance, power supply and current driver.

The prototype presented and assessed in this paper uses a single linear spring to hold the stoppers against the link. Though this design can result in some non-linearities in the spring induced moment, this design was selected to improve the compactness of the design. Task-specific requirements, however, can be addressed with small modifications to the design. For example, torsion springs can be used to improve moment linearity or elastic non-ferrous materials can potentially be used to allow integration into devices which work in environtments non conductive systems are needed (e.g. systems which work within the bore of a magnetic resonance imaging scanner). Also, miniature electromagnetic brakes were integrated into this prototype due to their simple operation and small price tag, but any other type of brakes can be used to activate stoppers with minor changes to the hardware design.

Finally, the weight of the current prototype unit is $467.1 \mathrm{~g}$, which is not ideal.- However, most of the weight is in the body of the prototype, while less than a half is taken by the miniature brakes (94g each) and thus structural optimization of the body should result in a significant reduction in weight without loss of performance.

\section{F. Mathematical Model of the Brake}

It can be seen through the work presented in this paper that the novel programmable brake can be classified as a new type of part-locking mechanism. This section aims to summarise its features by means of a mathematical model.

Assuming that the braking torque of the stoppers $A$ and $B$ can be varied in a range of 0 to 1 , where 0 is OFF and 1 is maximum torque, then the reaction torque as a function of the power of the stoppers $A$ and $B$, and the shaft angular velocity $\omega$ can be written as:

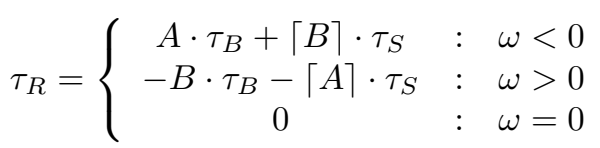




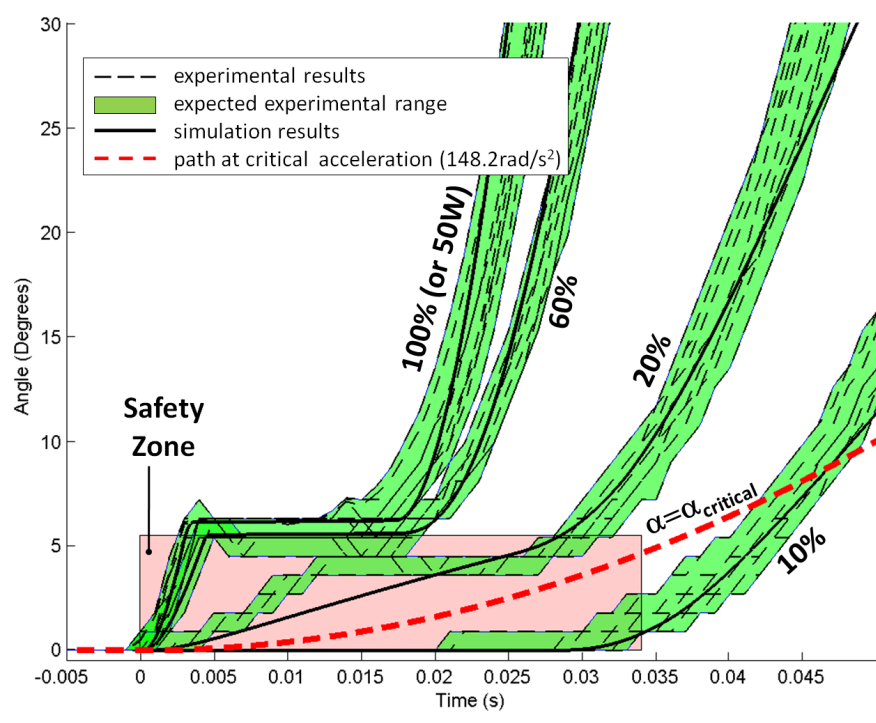

Figure 7: Simulated vs. experimental results

where $\tau_{R}$ is the reaction torque of the programmable brake, $\tau_{B}$ is the maximum friction torque produced by one brake and $\tau_{S}$ is the torque due to the preloaded spring.

These equations highlight that motion away from an actuated stopper would result in a reaction torque due to the spring $\left(\tau_{S}\right)$. In these equations, the spring induced torque $\left(\tau_{S}\right)$ can be simplified to a constant value, but, if a more accurate estimation is required, its value can be estimated from Eq. 4. Finally, the analysis of these equations shows that, by varying the power of the stoppers $\mathrm{A}$ and $\mathrm{B}$, the four state programmability of the shaft, as presented in Table I, can be achieved.

\section{Conclusion}

Passive mechanisms have great potential in human-machine interfaces and especially in medical applications where safety is of utmost importance. Existing designs lack simplicity and therefore are seldom used. In contrast, this paper presents a new type of programmable rotary brake for use in manmachine interfaces, which can be programmed into four states, is modular, has a simple structure, can be manufactured to be cost effective, and does not require any explicit force measurements when implemented into a device. Potential applications for the brake include haptic devices for rehabilitation (when large reaction forces are required to strengthen muscolar function), programmable end stops for industrial robots (which would prevent motion in an undesired direction in the event of a mulfunction) and virtual reality training systems, where the large reaction torques generated by the brake would enable stiff collisions (e.g. tool on bone) to be reproduced with ease and without endangering the user.

An early embodiment of the concept was presented in [23], where aspects of the programmable brake were discussed, together with an early set of experimental results which demonstrated the viability of the concept. This first proofof-concept prototype was bulky, not modular and employed custom-made brakes to operate the stoppers. A number of shortcomings, as well as areas which would benefit from analytical modeling and performance characterization, were also identified, which are the focus of this paper. Here, a novel modular spring-based design is proposed and, since the constrained shaft is an integral part of the programmable brake, integration into a general-purpose mechanical system is substantially simplified.-

The mechanical characteristics of the part-locking system can be grouped under two headings: brake-specific and mechanism-specific parameters.. Parameters which are typical of brakes, such as maximum reaction torque and activation time, are associated with the commercial brakes used in a specific embodiement and can therefore be extracted from manufacturer supplied datasheets). Mechanism-specific parameters relate to the performance of the programmable brake mechanism. Two - key performance parameters, the critical acceleration and the reaction torque ratio, were identified and derived both mathematically and through simulation. The existance of a "critical acceleration" was also verified experimentally, which was found to be $148.2 \mathrm{rad} / \mathrm{s}^{2}$ for the current prototype. Also, the maximum torque required to move away from the activated stopper was found to be $23.8 \mathrm{~m} \mathrm{Nm}$, which is about $2.6 \%$ of the maximum reaction torque of $0.9 \mathrm{Nm}$ for the miniature brake used. Finally, the equations derived in Section X capture the functional properties of this novel concept, which are different from other types of braking system (e.g. brakes or clutches). These can be used to simplify the modeling of any device where the part-locking mechanism is to be incorporated into the design.

To achieve part-locking restrictions, a suitable control scheme must be applied and three simple strategies are outlined: velocity control, position control and a more advanced control scheme based on both position and speed measurements. It is thus possible to control the brake in four states, while maintaining a simple and cost effective approach. The validity and potential problems of these control schemes were not evaluated in the scope of this paper and will be addressed in the scope of future work, where the programmable brake will be incorporated into a multi degree-of-freedom haptic display. Also, futher evaluation of the performance-parameters would require application-specific setup and will thus be adressed in the context of future work.-

The prototype presented in this paper uses commerciallyavailable brakes to operate the stoppers and therefore benefits from the advantages associated with commercial products , such as a high torque-to-size ratio, fast response, power efficiency, zero backlash, long life and reduced maintenance. However, a number of alternative braking strategies could be adopted with minor mechanical adjustments, for application where other brake types have an advantage. Due to its cylindrical, modular shape, the current design can also be considered for use instead of a motor in impedance-controlled haptic devices, where passivity is important.- As such, this novel programmable brake represents a marked improvement over existing part-locking designs and should be considered for future man-machine interfaces. Developing an interface based on the programmable brakes and evaluating its performance would be in the scope of future work. 


\section{REFERENCES}

[1] Magneta gmbh co kg. http://www.magneta.de.

[2] P. Akella, M. Peshkin, E. Colgate, W. Wannasuphoprasit, N. Nagesh, J. Wells, S. Holland, T. Pearson, and B. Peacock. Cobots for the automobile assembly line. Ieee International Conference on Robotics and Automation, (1):728-733, 1999.

[3] J. Bell, S. Bolanowski, and M. H. Holmes. The structure and function of pacinian corpuscles - a review. Progress in Neurobiology, 42(1):79-128, 1994.

[4] J. Blake and H. B. Gurocak. Haptic glove with mr brakes for virtual reality. IEEE/ASME Transactions on Mechatronics, 14(5):606-615, 2009.

[5] J. E. Colgate, W. Wannasuphoprasit, and M. A. Peshkin. Cobots: Robots for collaboration with human operators. In K. Danai, editor, ASME; Dynamic Systems and Control Division, pages 433-440, Atlanta; GA, 1996. Asme.

[6] B. Davies, M. Jakopec, S. J. Harris, F. Rodriguez y Baena, A. Barrett, A. Evangelidis, P. Gomes, J. Henckel, and J. Cobb. Active-constraint robotics for surgery. Proceedings of the Ieee, 94(9):1696-1704, 2006.

[7] H. Davis and W. Book. Torque control of a redundantly actuated passive manipulator. Proceedings of the American Control Conference, 2:959963, 1997

[8] Edward Alexander Dibble. A novel haptic system for use in training knee arthroscopy. PhD thesis, Imperial College, 2005.

[9] R. J. Farris and M. Goldfarb. Design of a multidisc electromechanical brake. IEEE/ASME Transactions on Mechatronics, $\mathrm{PP}(99)$ )1-9. (Accepted for publication).

[10] C. Francois, K. Oussama, and B. Charles. A hybrid actuation approach for haptic devices, 2007. 1264816 367-372.

[11] M. Gogola and M. Goldfarb. Design of a pzt-actuated proportional drum brake. Ieee-Asme Transactions on Mechatronics, 4(4):409-416, 1999.

[12] A. H. C. Gosline and V. Hayward. Eddy current brakes for haptic interfaces: Design, identification, and control. Ieee-Asme Transactions on Mechatronics, 13(6):669-677, 2008.

[13] D. Grant. Two new commercial haptic rotary controllers, 2004.

[14] B. Hannaford and J. H. Ryu. Time-domain passivity control of haptic interfaces. Ieee Transactions on Robotics and Automation, 18(1):1-10, 2002.

[15] N. Hungr. Haptic emulation of hard surfaces with applications to orthopaedic surgery. Msc, The University of British Columbia, 2008.

[16] T. B. Kwon and J. B. Song. Force display using a hybrid haptic device composed of motors and brakes. Mechatronics, 16(5):249-257, 2006.

[17] B. Liu, W. H. Li, P. B. Kosasih, and X. Z. Zhang. Development of an mr-brake-based haptic device. Smart Materials and Structures, 15:19601966, 2006.

[18] P. Lum, D. Reinkensmeyer, R. Mahoney, W. Z. Rymer, and C. Burgar Robotic devices for movement therapy after stroke: current status and challenges to clinical acceptance. Top Stroke Rehabil, 8(4):40-53, 2002.

[19] Louis B. Rosenberg. Method and apparatus for providing passive force feedback to human-computer interface systems, 1998. Patent : 5767839, United States.

[20] M. Russo and A. Tadros. Controlling dissipative magnetic particle brakes in force reflective devices American Society of Mechanical Engineers, Dynamic Systems and Control Division (Publication) DSC. 1992.

[21] O. Schneider, J. Troccaz, O. Chavanon, and D. Blin. Padyc: A synergistic robot for cardiac puncturing. Ieee International Conference on Robotics and Automation, 3:2883-2888, 2000

[22] D. K. Swanson, E. Romagna, W. J. Book, and A. Barraco. Influence of actuator dynamics on passive haptic interface performance. Ieee Asme International Conference on Advanced Intelligent Mechatronics Proceedings, pages 440-445, 1999.

[23] Y. Tenzer, B. Davies, and F. Rodriguez y Baena. Programmable differential brake for passive haptics. Journal of Robotics and Autonomous Systems, 58:249-255, 2009.

[24] C. Thorne, M. Yim, N. Skorodinski, H. Tipton, and T. Van Schoyck. Brake design for dynamic modular robots. 2010 IEEE International Conference on Robotics and Automation, May 3-8, 2010, Anchorage, Alaska, USA, 2010.

[25] M. V. Weghe, B. Dellon, S. Kelly, R. Juchniewicz, and Y. Matsuoka. Demonstration of a large dissipative-haptic environment. Symposium on Haptics Interfaces for Virtual Environment and Teleoperator Systems 2006, Proceedings, pages 329-330 538, 2006. 